\title{
Human Factors in Tele-inspection and Tele-surgery: Cooperative Manipulation under Asynchronous Video and Control Feedback
}

\author{
James M. Thompson ${ }^{1,2}$, Mark P. Ottensmeyer ${ }^{1}$, and Thomas B. Sheridan ${ }^{1}$ \\ ${ }^{1}$ Human Machine Systems Laboratory, Massachusetts Institute of Technology \\ ${ }^{2}$ Department of Anesthesia and Critical Care, Massachusetts General Hospital and Harvard \\ Medical School
}

\begin{abstract}
A telesurgical model was developed for simulation experiments evaluating cooperative manipulation between a paramedic local to the patient and a physician operating through a telerobot. In this study we tested the hypothesis that sending the signals from a remote telesurgical setup asynchronously (sending the telemanipulator signals ahead of the video signals, which were delayed because of the time it took for compression / decompression) improve controller stability and favorably affect task performance by the medical team. We found essentially no difference in task performance between the synchronous and asynchronous transmission of the telesurgical signals when the physician operated the laparoscope and the assistant operated the laparotomy tools. But with asynchronous transmission we found a significant improvement (31\% to 60\%) in task completion time when the physician operated any of the laparotomy tools.
\end{abstract}

\section{Introduction}

The purpose of this project is to evaluate telesurgery from a human factors engineering viewpoint. In particular it focuses on two interrelated problems:

1). Cooperative manipulation between a paramedic local to the patient and a physician operating through a telerobot.

2). Coping with time delay in both visual and force feedback.

In our first series of experiments, using synchronously transmitted video and telemanipulator signals, we found that the time delay made operation of the laparoscopic tools by the surgeon extremely difficult.

In the experiments reported here we asked the question: Does sending the signals from a remote telesurgical setup asynchronously (sending the telemanipulator signals ahead of the video signals, which were delayed because of the time it took for compression / decompression) improve controller stability and favorably affect task performance by the medical team.

Though the use of communication systems in medicine is not new, the use of a high bandwidth audio and video link has recently been applied to the diagnosis and treatment of the patient. Still, there are numerous situations where the bandwidth is not sufficient for the simultaneous transmission of teleoperator control signals, real time video signals and audio signals. In such a case, it is common to employ CODEC (compression and decompression) boards for the transmission of the video signals. Standard videoconferencing equipment over ISDN and fractional T1 phone lines currently use this technology for video conferencing calls. Video compression and 
decompression imposes a time delay, and combined with the time delay imposed by the communication system produces a total time delay that adversely affects the task completion time of currently proposed telesurgical systems.

Investigators have shown that teleoperator users start to use a "move and wait" strategy as the closed loop time delay increases above 0.1 seconds $[1,2]$. In our first series of experiments, we found that the synchronous transmission of video and telemanipulator signals (our model of $600 \mathrm{~ms}$ video delay plus a $600 \mathrm{~ms}$ communication delay) resulted in a total round trip time delay that seriously affected task performance time and resulted in a "move and wait" strategy when the surgeon operated the laparoscopic tools. In this current set of experiments we explore the hypothesis that sending the signals from a remote telesurgical setup asynchronously would improve controller stability and possibly favorably affect task performance by the surgical team.

\section{Methods}

The experimental system is presented in Figure 2.1 and described below.

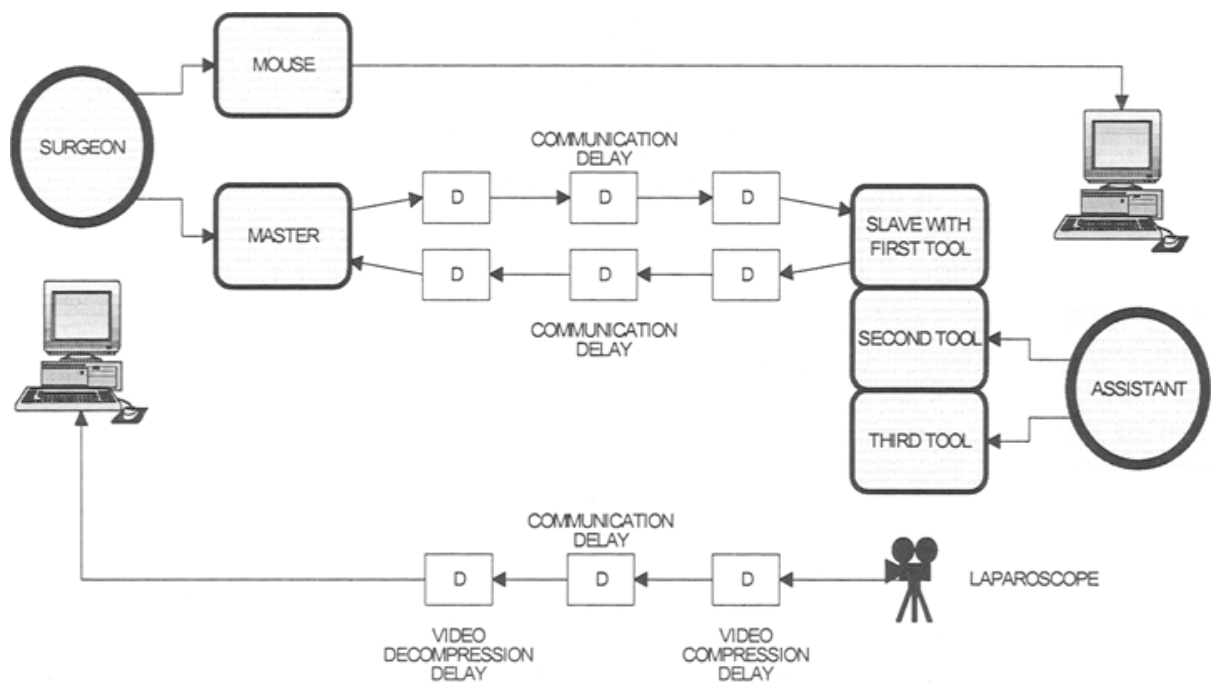

Figure 2.1 Telesurgical System

\subsection{Telemanipulator and Tools}

The telemanipulator and tools are important in enabling the surgeon to diagnose and treat the trauma patient in the remote environment. One needs to be able to control a laparoscope for viewing, and be able to manipulate a hemostat (to be used as a blunt probe or to grasp intra-abdominal contents), scissors and a gripper / clip applicator (for clamping the bleeding vessel).

\subsection{Telesurgical System: Time Delay}

In a telesurgical system one is faced with two types of time delays: (1) delay associated with the transmission of the signals from the teleoperator over a 
communication link, and (2) the delay due to the video compression and decompression because of a communication bandwidth limit.

The transit time for signals sent over communication channels depend on the medium (e.g. ISDN, satellite) and the traffic over that medium. With an ISDN setup, the typical round trip delays are in the order of $600 \mathrm{~ms}$ [3]. The distance can also be a factor. Researchers reported a 1.4 second round trip delay in a teleoperator connection between Tokyo and Washington, D.C. [4].

There is also a delay associated with the video compression and decompression needed when unlimited bandwidth is not available. Current video conferencing equipment that uses ISDN lines requires approximately $300 \mathrm{~ms}$ for the video compression and subsequent decompression for one way transmission. The components of these delays are shown in Figure 2.1.

\subsection{Experimental Apparatus}

Our experimental set-up is intended to model the telesurgical system described in the previous section. In particular, our set-up for the asynchronous experiments is as shown in Figure 2-1. The components were designed and constructed during the initial stages of our project and explained in more detail in our previous report, Human Factors in Tele-Inspection and Tele-Surgery: Cooperative Manipulation and Time Delay, dated 9 October 1995.

In order to make the working environment as realistic as possible, we used laparoscopic tools of the type currently being used in the operating room which were modified for our use. The tool handle was detached from the tip and attached to the master manipulator. Because the PHANToM telemanipulator had only three translational degrees of freedom, a single rotational degree of freedom (around the tool axis) was mechanized. For the slave manipulator, the tool tip was modified to provide a roll axis and is attached to the slave telemanipulator using the gimble wrist end of the telemanipulator. The test surgeon operated these tool handles just as he would in normal laparoscopic surgery.

The telemanipulators used were paired PHANToM manipulators that were initially developed as a force-reflecting interface. One of the phantoms, operated by the test surgeon, was used as a master on which the tool handle was fixed. The second manipulator operated as a slave (to which the other end of the laparoscopic tool was attached). The control software used was developed as part of the project [5].

The audio / visual system consisted of microphones and headsets, cameras and displays and a two-way communications setup. Thus both audio and video were available to both the surgeon and assistant. We used two matched headphone / boom microphone systems connected through a pre-amp / amplifier to the audio delay boards. A CCD camera was used for the laparoscope.

We used a Prime Image A/V mainframe with two video delay boards and a 2 channel audio board to generate the audio and visual delays. This was connected to our camera, monitors and audio communication equipment and used to model the transmission line and video compression / decompression delays.

The video images were recorded with a Video Hi-8 recorder and routed to the two video monitors and VHS recorder through a Silicon Graphics Galileo video board. 
We used the same surgical laparoscopic simulators designed and constructed for our first series of experiments. The simulator used for our experiments was modeled after laparoscopic training simulators $[6,7]$ which has four surgical incision sites for trocar insertion and has a clear side wall for recording the experiments with a camcorder.

In our experimental setup, the surgeon operated a mouse pointer that was superimposed over the image from the surgical site and was used by the surgeon as a visual tool to direct the actions of the assistant. This video overlay was generated by a Silicon Graphics Indigo2 Extreme workstation using the Galileo video card.

\subsection{Experimental Surgical Tasks Used}

\section{Grasp and Transfer Experiments}

This experiment evaluated the ability of the surgical team to control the hemostat and to work together to perform a task. The performance time was defined as the time it takes for the surgeon - assistant team to transfer 6 clips from one location to the other and back.

\section{Hemostasis}

In this experiment we evaluated the use of the clip applicator / hemostat and the scissors by the surgical team (surgeon and assistant). We started with a model of the neurovascular bundle (a nerve, artery and vein usually travel together) made up of tan, blue and red rubber bands. The task completion time was measured as the time it took the team to correctly place the hemostat / clipper completely around the related vessel (with the exclusion of adjacent structures), clip off the vessel, and then to cut the redundant vessel with the scissors.

\subsection{Experimental Subjects}

The test surgeon throughout these preliminary experiments was an experienced emergency room physician and anesthesiologist. The assistants were engineering graduate students. These were the same individuals that were tested in the first set of experiments run the previous year.

\subsection{Data Recording}

The experiments were recorded to a VCR deck using a high fidelity Sony Camcorder. The first author later reviewed these videotapes and the task completion times were recorded for each of the individual experiments.

\subsection{Experimental Design: Synchronous and Asynchronous Transmission}

In our first set of experiments we buffered the telemanipulator signals while the video signal was being processed, then sent both the video and telemanipulator signals simultaneously (synchronous) over the transmission link. In our second set of experiments we immediately sent the telemanipulator signals over the transmission link without waiting for the video signals (which needed 0.6 second round trip for the video compression-decompression) which resulted in asynchronous transmission of video and telemanipulator signals. 


\section{Results}

The results for the individual tool combinations for each experiment are presented in Figures 3.1 to 3.4 and discussed in the following sections of this paper.

In general, we found that when the surgeon operated the laparoscope there was a slight improvement in task completion time when the task was performed with asynchronous (as compared to synchronous) video and telemanipulator feedback signals. When the surgeon used any of the other tools, we found a dramatic improvement in task completion time when the signals were sent asynchronously, for both the 0.6 second and 1.2 second delays.

\subsection{Grasp and Transfer Experiments}

There were two physician / assistant tool combinations for this experiment. In the first scenario, the physician operated the scope through the teleoperator while the assistant operated the two hemostats. In the second scenario, the surgeon operated one of the hemostats while the assistant operated the laparoscope and the other hemostat. As in our first series of experiments, we found that task completion time is lowest (better performance) when the surgeon operated the laparoscope rather than operating one of the hemostats. We found this to be the case in all six experiments in which we varied the time delays for both the video and telemanipulator signals.

In our second set of experiments we studied the effect of asynchronous video and telemanipulator signal transmission on task completion time with various time delays. The results show a significant improvement in task completion time with asynchronous (as compared to synchronous) signal transmission. The results for the experiments with no transmission time delays are shown in Figure 3.1.

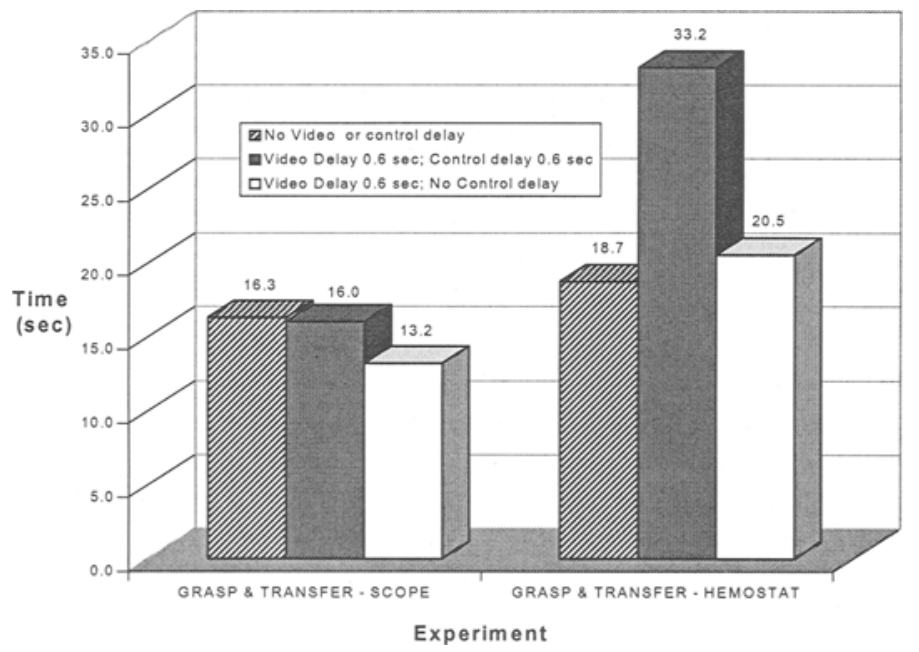

Figure 3.1. Grasp and Transfer Experiments: Performance times for asynchronous and synchronous signals with no transmission time delay. ("Grasp \& Transfer - Scope" means that the surgeon operated the laparoscope in the Grasp \& Transfer experiment) 
The results for a transmission time delay of 0.6 seconds are presented in Figure 3.2 .

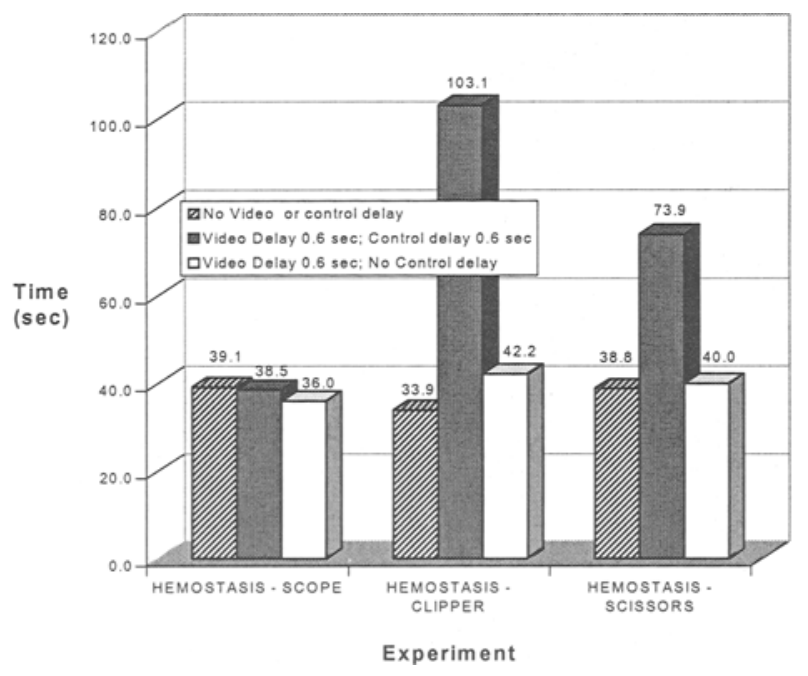

Figure 3.2. Grasp and Transfer Experiments: Performance times for asynchronous and synchronous signals with a 0.6 second transmission time delay. ("Hemostasis - Scope" means that the surgeon operated the laparoscope in the Hemostasis experiment)

\subsection{Hemostasis Experiments}

There were three physician / assistant tool combinations for this experiment, a laparoscope, clipper / hemostat, and a scissors. In the first scenario, the physician operated the scope through the teleoperator, while the assistant operated the clipper and the scissors. In the second scenario, the surgeon operated the clipper / hemostat while the assistant operated the laparoscope and the scissors. In the third scenario the physician operated the scissors while the assistant operated the laparoscope and the clipper. As in our first series of experiments, task completion time is lowest (better performance) when the surgeon operated the laparoscope rather than operating one of the other tools. We found this to be the case in all six experiments in which we varied the time delays for both the video signals and the telemanipulator signals.

In our second set of experiments we studied the effect of asynchronous video and telemanipulator signal transmission on task completion time with various time delays. Our results, which are presented below for each time delay, also showed a significant improvement in task completion time with asynchronous (as compared to synchronous) signal transmission, except when the physician operated the laparoscope. The results for the experiments with no transmission time delays are shown in Figure 3.3. The results for a transmission time delay of 0.6 seconds are presented in Figure 3.4.

\section{Discussion}

In this second series of experiments in our telesurgery project we attempted to 


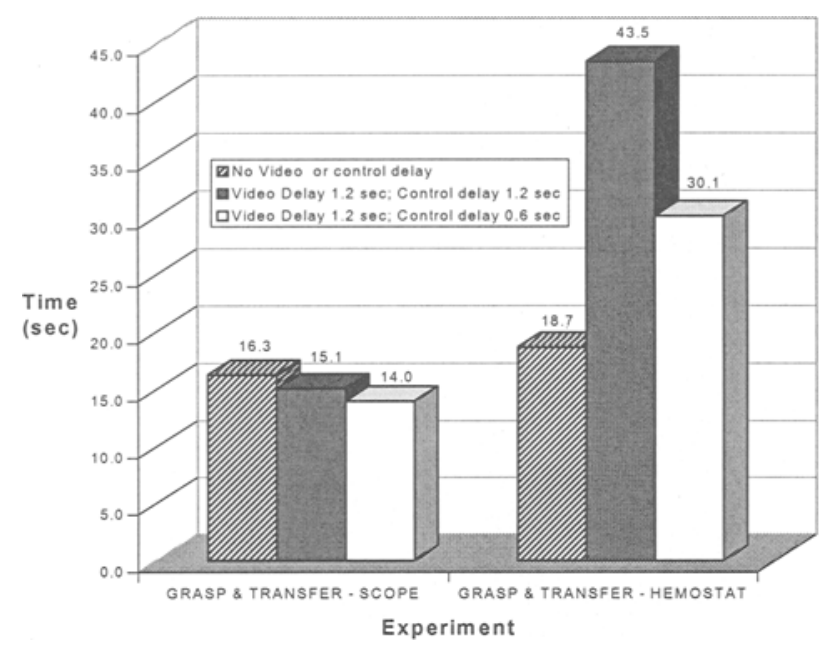

Figure 3.3. Hemostasis Experiments: Performance times for asynchronous and synchronous signals with no transmission time delay. ("Grasp \& Transfer - Scope" means that the surgeon operated the laparoscope in the Grasp \& Transfer experiment)

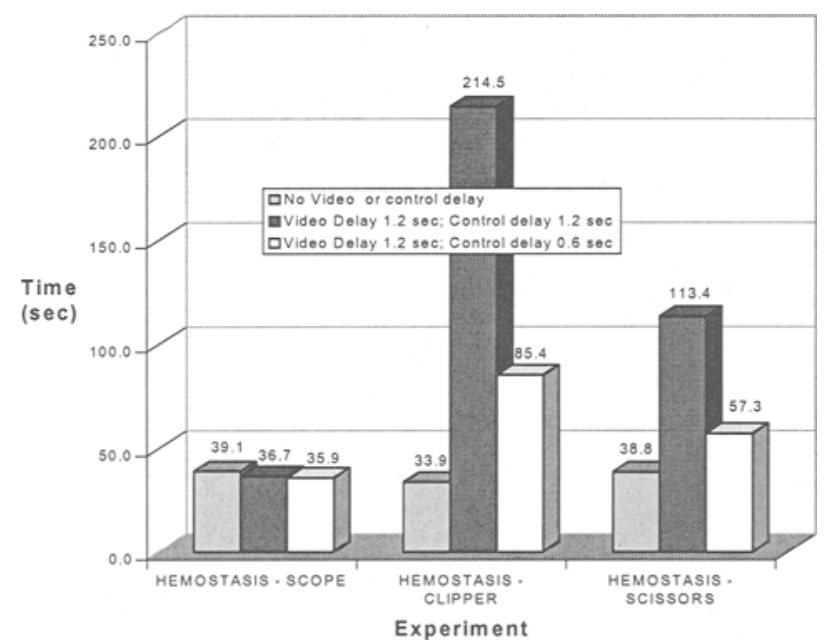

Figure 3.4. Hemostasis Experiments: Performance times for asynchronous and synchronous signals with a 0.6 second transmission time delay. ("Hemostasis - Scope" means that the surgeon operated the laparoscope in the Hemostasis experiment)

test the hypothesis that sending the signals from a remote telesurgical setup asynchronously (sending the telemanipulator signals ahead of the video signals, which were delayed because of the compression / decompression it required) would 
improve controller stability and favorably affect task performance by the surgical team.

We had interesting results. There was essentially no difference in task performance between the synchronous and asynchronous transmission of the telesurgical signals when the physician operated the laparoscope. But we found a significant improvement $(31 \%$ to $60 \%$ ) in task completion time when the physician operated any of the laparotomy tools with asynchronous transmission when compared to synchronous transmission.

The results when the physician operated the laparoscope can be easily explained. Degradation in team performance resulting from the effects of time delay (controller instability) is reduced by several factors. First, because the laparoscopic output is an entire field, the person controlling the laparoscope needs to be less sophisticated. Secondly, because the surgeon knows the contents of the surgical field, and what task sequence needed to be performed, he/she is more efficient in minimizing movements (stabilizing field of view) of the laparoscope. In essence, the control of the laparoscope was fairly passive as compared to the operation of the laparoscopic tools.

The significant improvement in task completion time (for asynchronous as contrasted to synchronous transmission) when the surgeon operated the laparoscopic tools can be explained by looking at the effect of time delay on stability of the telemanipulator system. The main advantage with asynchronous transmission probably resulted from the increased stability of the telerobotic manipulator because of the decreased time delay seen by the controller. In the case of synchronous transmission (Figure 2.1), since the telemanipulator signals were sent with the video signals, the controller had an additional delay due to the time it took to compress and then decompress the video signals. The controller delay was

$$
\mathrm{T}_{\mathrm{d}}=\mathrm{D}_{\mathrm{c}}+\mathrm{D}+\mathrm{D}_{\mathrm{d}}
$$

where: $T_{d}$ is the total delay seen by the controller

$\mathrm{D}_{\mathrm{c}}$ is the delay due to video compression

$\mathrm{D}$ is the delay due to signal transmission (e.g. phone line)

$\mathrm{D}_{\mathrm{d}}$ is the delay due to video decompression

In the case of asynchronous transmission (Figure 2.1), the telemanipulator signal was sent ahead of the video signal and the controller delay was then:

$$
\mathrm{T}_{\mathrm{d}}=\mathrm{D}
$$

where: $T_{d}$ is the total delay seen by the controller

$\mathrm{D}$ is the delay due to signal transmission (e.g. phone line)

We see that the controller had a smaller time delay in the asynchronous mode, which made it more stable and easier for the physician to operate the tools.

The main disadvantage with asynchronous transmission was that the operator did not receive the force feedback information at the same that he received the video input. The results of our experiments seem to suggest that the improvement in performance because of the more stable controller more than offset the degradation in 
performance due to the asynchronous force feedback and visual image seen by the physician.

\section{Conclusions}

In this second series of experiments in our telesurgery project we attempted to test the hypothesis that sending the signals from a remote telesurgical setup asynchronously (sending the telemanipulator signals as quickly as possible, ahead of the video signals, which are necessarily delayed because of the compression / decompression required) improves controller stability and favorably affects task performance.

We found essentially no difference in task performance between the synchronous and asynchronous transmission of the telesurgical signals when the physician operated the laparoscope and the assistant operated the laparotomy tools. But with asynchronous transmission we found a significant improvement $(31 \%$ to $60 \%)$ in task completion time when the physician operated any of the laparotomy tools.

This is an important finding that can be applied to any situation which involves the cooperative actions of an expert operating a remote telemanipulator system (that is limited by bandwidth considerations) and a non-expert person operating some tools at the local site (where the telemanipulator slave is working).

\section{References}

1. Ferrell, W. R. and Sheridan, T. B.: Supervisory control of remote manipulation. IEEE Spectrum 4, no. 10, (1967):81-88.

2. Black, J.: Factorial study of remote manipulation with transmission time delay. Master's thesis, MIT, Engineering Projects Lab, Department of Mechanical Engineering, Dec. 1970.

3. Zydacron, Inc.: ZydApp2 User's Guide for software release 1.1, February:87.

4. Mitsuishi, M., Watanabe, T., Nakanishi, H., Hori, T., Watanabe, H., and Kramer B.: A telemicro-surgery system with co-located view and operation points and a rotational-forcefedback-free master manipulator. Second Annual International Symposium on Medical Robotics and Computer Assisted Surgery: (1995):111-118.

5. Hu, J., Ren, J., Thompson, J. M., and Sheridan, T. B.: Fuzzy sliding force reflecting control of a telerobotic system. Proceedings of IEEE Conference on Fuzzy Systems, September 1996.

6. Bailey, R. W., Imbembo, A. L., and Zucker, K. A.: Establishment of a laparoscopic cholecystectomy training program. The American Scientist: 57(4), (1991):231-236.

7. Muntzer, M.: A cheap laparoscopic surgery trainer. Annals of the Royal College of Surgeons of England: 74:256-257. 6. Bailey, R. W., Imbembo, A. L., and Zucker, K. A. (1991). Establishment of a laparoscopic cholecystectomy training program. The American Scientist: 57(4), (1992):231-236. 